\title{
Experimental observation of negative differential resistance from an InAs/ GaSb interface
}

\author{
D. A. Collins, E. T. Yu, Y. Rajakarunanayake, J. R. Söderstrom, ${ }^{\text {al }}$ D. Z.-Y. Ting, \\ D. H. Chow, and T. C. McGill \\ Thomas J. Watson, Sr. Laboratory of Applied Physics, California Institute of Technology, \\ Pasadena, California 91125
}

(Received 23 March 1990; accepted for publication 5 June 1990)

We have observed negative differential resistance at room temperature from cevices consisting of a single interface between $n$-type InAs and $p$-type GaSb. InAs and GaSb have a type II staggered band alignment; honce, the negative differential resistance arises from the same mechanism as in a $p^{+}-n^{+}$tunnel diode. Room-temperature peak current densities of $8.2 \times 10^{4}$ $\mathrm{A} / \mathrm{cm}^{2}$ and $4.2 \times 10^{4} \mathrm{~A} / \mathrm{cm}^{2}$ were measured for structures with and without undoped spacer layers at the heterointerface, respectively.

Feterojunction resonant tunneling diodes have been extensively studied since their original proposal ${ }^{l}$ and demonstration. ${ }^{2}$ The majority of these devices kave been grown in $\mathrm{Al}_{x} \mathrm{Ga}_{1} \ldots \mathrm{As} / \mathrm{GaAs}$, in which electrons resonantly tunnel through quasi-bound states in the GaAs conduction band giving rise to negative differential resistance (NDR). Recently, NDR has been proposed and observed in the nearly lattice-matched InAs/GaSb/AlSb system. ${ }^{3-7}$ In these interband tunnel devices, the mobile charge carriers tumel between the conduction band of one material and the valence band of an adjacent material. These novel devices, as well as being of scientiffc interest, possess electrical properties which may have practical technological consequences: large peak-to-valley current ratios (PVRs) ${ }^{4.5}$ large peak current densities, ${ }^{6,7}$ and theoretical predictions of very high device speeds. 8,9

In this letter we report the experimental observation of NDR in a device consisting of a single InAs/GaSb interface. The devices showed NDR at room temperature with peak current densities of $8.2 \times 10^{4} \mathrm{~A} / \mathrm{cm}^{2}$ and $4.2 \times 10^{4} \mathrm{~A} / \mathrm{cm}^{2}$ for structures with and without undoped spacer layers on each side of the heterointerface, respectively. These peak currents represent an average over 25 randomly chosen devices, with a standard deviation in the measured value of $\sim 0.5 \times 10^{4}$ $\mathrm{A} / \mathrm{cm}^{2}$. The room-temperature PVR ranged from $1.1: 1$ to 1.5:1 with the doped interface giving the higher value. In 1977 Sakaki et al. measured the current-voltage (I-B) curves of a structure with an $\mathrm{In}_{0.84} \mathrm{Ga}_{0.16} \mathrm{As} / \mathrm{GaSb}_{0.9} \mathrm{As}_{0.2}$ interface, but did not observe NDR. ${ }^{10}$ We believe that this was due to the fact that the mesas they fabricated had a device area over 250 times larger than the ones reported here. Because of the large current densities found in these structures (which ate comparable to those measured by Sakaki $e t$ al.) it is essential to fabricate small area devices so that ohmic heating due to contact and parasitic series resistance does not destroy the device before it can be biased into the NDR region.

\footnotetext{
a) Present address: Chalmers Giviversity of Technology, Department of Physies, \$-41296 Göteborg, Sweden.
}

The sampies studied were grown on (100) GaAs substrates in a Perkin-Elmer 430 molecular beam epitaxy systerm. A five period, 1 monolayer/l monolayer, $\operatorname{In}_{0.7}, \mathrm{Ga}_{0.3} \mathrm{As} /$ GaAs superlattice was grown at the GaAs/InAs interface to reduce the number of threading dislocations in the subsequent growth. ${ }^{11,12}$ Following the superlattice, $7000 \AA$ of InAs, Si-doped $n$-type $\left(n \sim 2 \times 10^{17} \mathrm{~cm}^{-3}\right)$, was grown at a rate of $1.0 \mu \mathrm{m} / \mathrm{h}$ at a substrate temperature of $460^{\circ} \mathrm{C}$. The growth was interrupted at this point and the temperature of the indium oven was lowered. Following this an additional $500 \AA$ of $n$-type InAs and $100 \AA$ of undoped InAs were grown at a rate or $0.5 \mu \mathrm{m} / \mathrm{h}$. The lower growth rate helps to smooth the growth surface and requires a smaller arseric flux, leading to less arsenic incorporation in the GaSb 1ayer. ${ }^{13}$ At the heterointerface the growth was interrupted for 2 min, while sitting in an arsenic fux, and the temperature of the arsenic cell was started down to its idle temperature. The substrate temperature was raised to $485^{\circ} \mathrm{C}$ during this interrupt and held there for the remainder of the growth. After an additional $5 \mathrm{~s}$ interrupt with the substrate in an antimony fiux, the growth of $100 \hat{A}$ of undoped $G a S b$ and $2000 \AA$ of $p$-type $G a S b$ was commenced at a rate of 0.5 $\mu \mathrm{m} / \mathrm{h}$. The GaSb was doped $p$ type with Si $\left(p \sim 5 \times 10^{18}\right.$ $\left.\mathrm{cm}^{-3}\right) \cdot{ }^{14} \mathrm{~A}$ second structure was grown in the same manner without the undoped spacer layers at the heterointerface.

The crystal's surface was monitored during the growth using reflection high-energy electron difraction (RHEED). The InAs (GaSb) surface showed a $2 \times 4$ (1 $\times 3)$ RHEED pattern indicating an As (So)-rich surface reconstruction. However, during the $5 \mathrm{~s}$ growth interrupt when the InAs surface sat in an Sb fiux the RHEED pattern changed to a $1 \times 3$ reconstruction similar to that of $\mathrm{InSb}$ indicating the formation of an $\operatorname{InAs}_{x} \mathrm{Sb}_{1 \ldots x}$ alloy at the heterointerface. We see no evidence of interdiffusion of the InAs and $\mathrm{GaSb}$ although we are pursuing more careful studies of this in order to find the optimal growth temperatures.

The structure was terminated with GaSb so that after processing, lateral conduction will be through the lower resistance InAs. Circular device mesas, $6 \mu \mathrm{m}$ in diameter, were formed using photolithography and a wet etch. Au/Ge was 


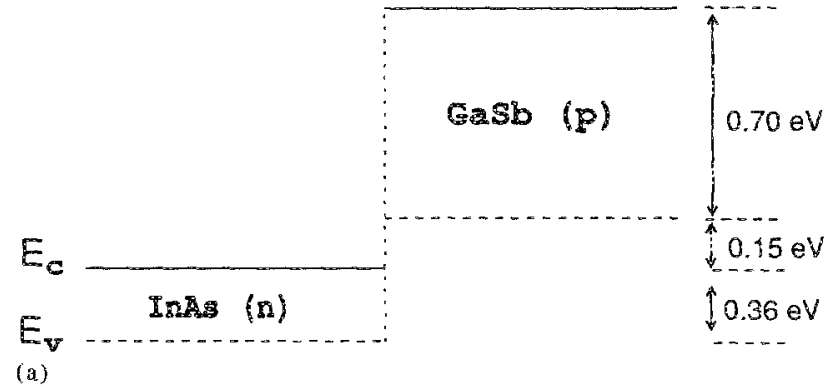

(a)

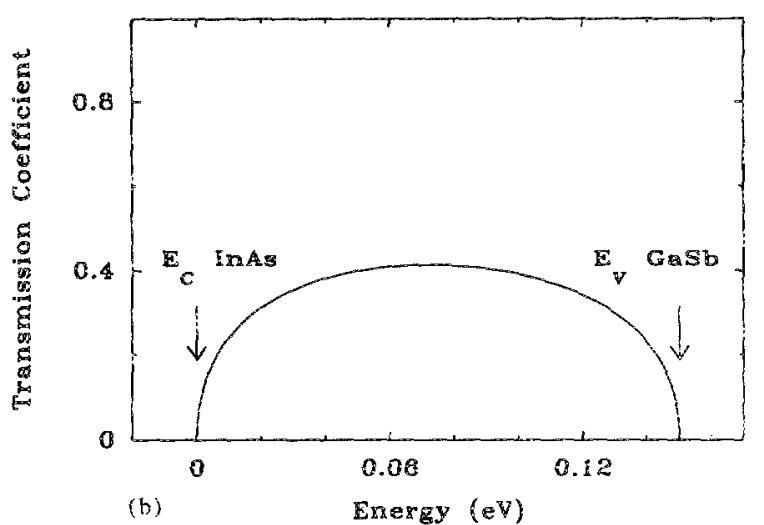

FIG. 1. (a) Schematic band diagram of the structure. (b) Transmission probability calculated with a two-band tight binding model.

used to make ohmic contact to the p-type GaSb. The etch was stopped in the InAs layer and $I-V$ curves were measured at room temperature and $77 \mathrm{~K}$ by probing the mesas with a thin gold wire.

In Figs. 1 (a) and 1(b) we show a band-edge diagram for the structure and the calculated transmission coefficient at flat band, respectively. The valence-band offset at the heterointerface is taken to be $0.51 \mathrm{eV} .^{15}$ The transmission probability is found from a two band model incorporating electrons and light holes. ${ }^{8}$ The calculated transmission coefficient does not have a Lorentzian line shape, showing that the device does not have a quasi-bound state, as in traditional resonant tunneling structures; however, the modulation of the structure's transmission coefficient as shown in Fig. $1(b)$ indicates that current conduction in the device is not merely ohmic in nature. The NDR observed in the structure is due to the GaSb band gap blocking the current channel in a manner similar to that of a forward biased, $p^{+}-n^{+}$ junction tunnel diode.

In Figs. 2 and 3 we show representative $I-V$ curves for the structures with and without the undoped spacer layers, respectively. Both devices show NDR at room temperature with peak current densities greater than $8.2 \times 10^{4}\left(4 \times 10^{4}\right)$ $\mathrm{A} / \mathrm{cm}^{2}$ for the structure with (without) undoped spacer layers at the heterointeriace. $I-V$ curves are shown at both $77 \mathrm{~K}$ and room temperature. The fact that the device's performance is virtually unchanged at the lower temperature indicates that thermionic emission plays oniy a minor role in the large valley current.

The valley currents in these structures are surprisingly large considering that they should be biocked by the GaSb

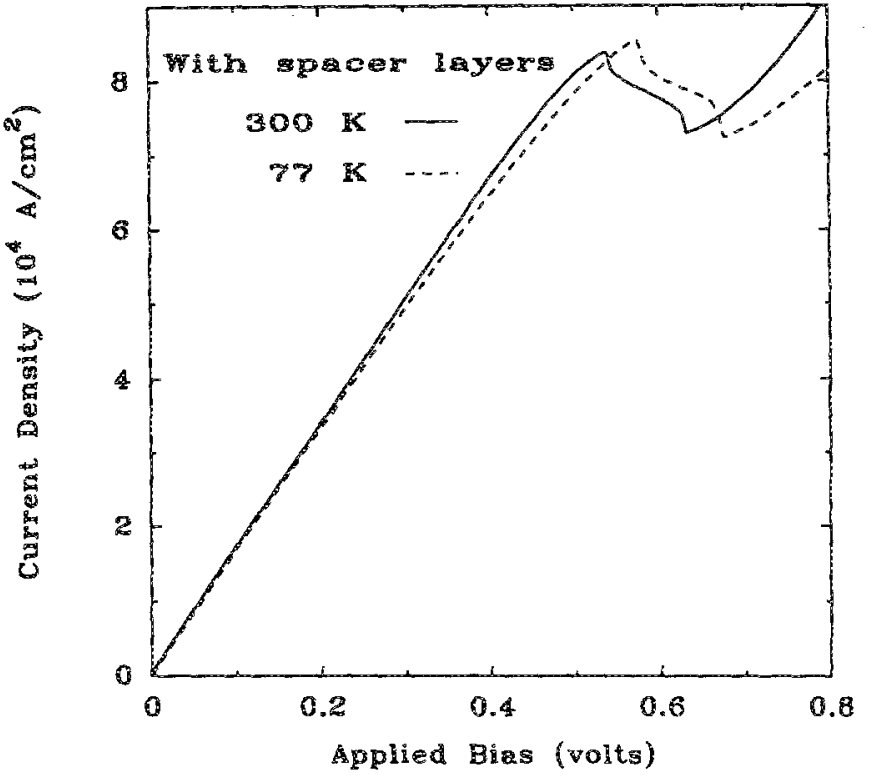

FIG. 2. Representative $I-V$ curves from the undoped interface. The solid line was taken at room temperature and the dashed line at $77 \mathrm{~K}$.

band gap. However, because there is not a true barrier between the $\ln \mathrm{As}$ conduction band and the GaSb valence band [see Fig. 1(b)] there is little to suppress inelastic processes from scattering electrons in the InAs conduction band into the GaSb valence band. It is interesting to note that in the $I-V$ curves shown in Figs. 2 and 3 the difierence between the peak current and the minimum valley current is approximately the same in both devices. The data seem to indicate that doping the device up to and through the interface reduces some of these inelastic currents.

Because the NDR observed in these devices is not associated with a quasi-bound state which may have a long life-

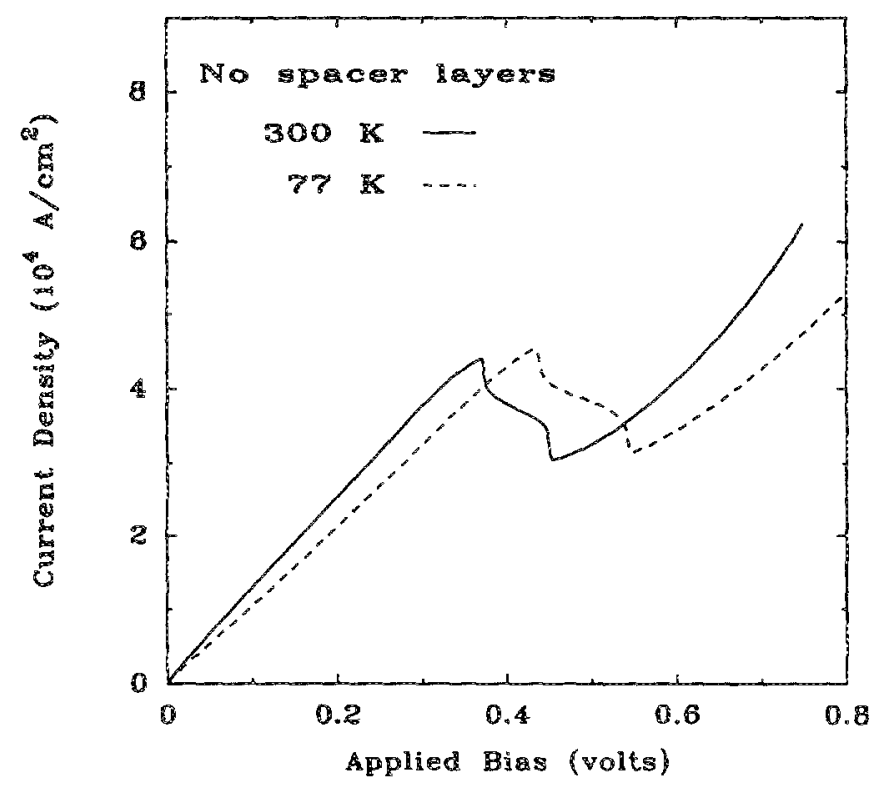

FIG. 3. Representative I- $V$ curves from the doped interface. The solid line was taken at room temperature and the dashed line at $77 \mathrm{~K}$. 
time, this device holds promise for the fabrication of high frequency oscillators. The intrinsic upper limit of this structure's oscillation frequency will be determined by the transit time across the heterointerface and the inherent frequency response of the materials. Furthermore, because this device can be grown without undoped spacer layers, limiting processes such as transit time delays across depleted regions which are important in conventional double-barrier structures will be eliminated in this device. ${ }^{\text {it }}$ For this structure to prove useful, however, it will be necessary to improve the PVR to 2 or greater. It may be possible to achieve this by optimizing the structure's doping profile or by tuning the band gaps and ofiset at the heterointerface by using $\mathrm{Al}_{u}, \mathrm{Ga}_{1-w} \mathrm{As}_{x} \mathrm{Sb}_{1-x} / \mathrm{In}_{y} \mathrm{Ga}_{1-y} \mathrm{As}_{z} \mathrm{Sb}_{1-z}$. Furthermore, it should be pointed out that there is an $\sim 0.6 \%$ lattice mismatch between InAs and Ga\$b. From this we estimate that the critical thickness for GaSb grown on InAs will be on the order of several hundred angstroms which is much less than the thickness of the GaSb epilayers reported on kere. Due to this, we anticipate misfit dislocations in the GaSb epilayers which may degrade device performance. By growing thinner layers we should be able to reduce or even eliminate the number of these interfacial misfit dislocations.

In summary we have observed NDR from an InAs/ $\mathrm{GaSb}$ interface. The peak current densities were $8.2 \times 10^{4}$ and $4.2 \times 10^{-4} \mathrm{~A} / \mathrm{cm}^{2}$ for undoped and doped interfaces, respectively. These current densities are comparabie to those found in conventional $\mathrm{Al}_{x} \mathrm{Ga}_{1}$. As/GaAs tunneling $\mathrm{d}$ odes, indicating that they may be potentially useful as highfrequency oscillators. The fact that the mobile charge carriers in these structures do not have to tunnel across any classically forbidden regions or traverse any depleted material further indicates that their intrinsic oscillation frequen- cies could be quite high. $I$-V characteristics for these devices are virtually identical at room temperature and $77 \mathrm{~K}$, which indicates that thermionic currents play a small role in their operation.

The authors would like to thank P. C. Sercel and M. K. Jackson for helpful discussions. This work was supported by the Office of Naval Research under contract No. NOOO14. $89-1-141$.

'R. Tsu and L. Esaki, Appl. Phys. Lett. 23, 562 (1973).

21. L. Chang, L. Esaki, and R. Tsu, Appl. Phys. Lett. 24, 593 (1974).

${ }^{3}$ M. Sweeny and J. Xu, Appl. Phys. Lett. 54, 546 (1989).

4. R. Söderström, D. H. Chow, and T. C. McGill, Appl. Phys. Lett. 55, 1094 (1989).

SL. F. Luo, R. Beresford, and W. I. Wang, Appl. Phys. Lett. 55, 2023 (1989).

${ }^{6} \mathrm{~K}$. Taira, I. Hase, and K. Kawai, Proceedings of the Seventh International Workshop on Future Electron Devices, Superlattice and Quantum Functional Devices, Ott 2-4, 1989, Toba, Japan (Plenum, New York, 1989), pp. 191-192.

DD. A. Collins, D. H. Chow, and T. C. McGill (unpublished).

${ }^{\text {DD. Z. }}$ Y. Ting, E. T. Yu, D. A. Collins, D. H. Chow, and T. C. McGill, I. Vac. Sci. Technol. B \&, \&10 (1990).

${ }^{9}$ J. R. Söderström, E. T. Yu, M. K. Jackson, Y. Rajakarmanayake, and T. C. McGili, $J$. Appl Phys. 68, 1372 (1990).

"H. Sakaki, L. L. Chang, R. Ludeke, Chin-An Chang, G. A. Sai-Halasz, and L. Esaki Appl. Phys. Lett. 31, 211 (1977).

"S. Kalem, J.-R. Chyi, and H. Morkoc, Appl. Phys. Lett. 53, 1648 (1988).

12 J. R. Söderström, D. H. Chow, and T. C. MeGili, Mater. Res. Soc. Symp. Proc. 145, 409 (1989).

${ }^{13}$ D. H. Chow, R. H. Miles, J. R. Söderström, and T. C. McGill, J. Vac. Sci. Technol $B 8,710(1990)$.

${ }^{14}$ T. M. Rossi, D. A. Collins, D. H. Chow, and T. C. McGill (unpublished) ${ }^{15}$ G. J. Gaultieri, G. P. Schwartz, R. G. Nuzzo, R. J. Malik, and J. F. Walker, J. Appl. Phys. 61, 5337 (1987).

${ }^{16}$ E. R. Brown, W. D. Goodhue, and T. C. L. G. Sollner, J. Appl. Phys. 64, $1519(1988)$ 